\title{
Tipos de prótesis sobre implantes en pacientes edéntulos planificados en la clínica dental docente de la Universidad Peruana Cayetano Heredia
}

Types of prosthesis on implants in patients edentulous planned in the teaching dental clinic of the Peruvian University Cayetano Heredia

José Antonio Balarezo Razzeto ${ }^{\text {1a,b,d;2,h }}$, Edinson Antonio Díaz Sarabia ${ }^{1 a, b, d, g ; 2, h}$, Fernando Ítalo Brignardello Cedrón 1,a,2,h, Carlos Alberto Paz Mayurí 1,a,f;;,,h, Claver Clemente Santos Escalante 1,a;,,h, Waldo Jesús Ronquillo Herrera ${ }^{1, a ; 2, \text {, ; (†) }}$, Roberto Leon Manco ${ }^{\text {1,a; ;,e }}$, Martín Quintana del Solar ${ }^{1, a, b, d ; 2,2, ~}$.

\section{RESUMEN}

Objetivo: Determinar la frecuencia de tipos de prótesis sobre implantes planificados en pacientes edéntulos en los servicios del Posgrado de Rehabilitación Oral e Implantología Oral Integral de la Clínica Dental Docente de la Universidad Peruana Cayetano Heredia durante los años 2016 y 2017. Material y métodos: La investigación fue descriptiva, retrospectiva, observacional y transversal, las variables: los tratamientos planificados, clasificándolos por género, edad, sede, servicio, tipo de prótesis sobre implantes, clasificación de edentulismo y zona edéntula. Se empleó la base de datos de todas las historias clínicas digitalizadas con presentación de caso planificada y aceptada por el docente tutor y acta de compromiso aceptada por el paciente. Resultados: Se encontraron 296 diversos tipos de prótesis sobre implantes, $68.58 \%$ en el sexo femenino, la edad promedio fue de 54,23 años, el mayor porcentaje entre los 60 y 69 años, la sede de San Isidro fueron planificados 61,15\%, en el servicio de RO 60,81\%, el mayor porcentaje de prótesis sobre implantes se realizó en el maxilar superior, el tipo de prótesis sobre implante con mayor porcentaje fue la prótesis unitaria $58,78 \%$, según clasificación de edentulismo se encontró el mayor porcentaje en el edéntulo parcial, el mayor porcentaje en el edentulismo total fue la prótesis híbrida con 59,46\% y en el edentulismo parcial fue la prótesis unitaria con un 66,80\%. Conclusiones: La mayor frecuencia fue para las prótesis unitarias, según género fue el femenino, según sede fue San Isidro y según servicio RO, según zona edéntula el maxilar superior, en edéntulos totales la prótesis híbrida y en edéntulos parciales la prótesis unitaria.

PALABRAS CLAVE: Arcada edéntula, arcada desdentada, prótesis dental, implantes dentales. 


\section{SUMMARY}

Objective: To determine the frequency of types of prostheses on planned implants in edentulous patients in the postgraduate services of Oral Rehabilitation and Integral Oral Implantology of the Teaching Dental Clinic of the Universidad Peruana Cayetano Heredia during the years 2016 and 2017. Materials and methods: The research was descriptive, retrospective, observational and transversal, the variables: the planned treatments, classifying them by gender, age, seat, service, type of prosthesis on implants, classification of edentulism and edentulous zone. The database of all the digitalized medical histories was used with the presentation of the planned case and accepted by the tutor and commitment certificate accepted by the patient. Results: We found 296 different types of prostheses on implants, $68.58 \%$ in the female sex, the average age was 54.23 years, the highest percentage between 60 and 69 years, the San Isidro headquarters were planned $61.15 \%$, in the service of RO $60.81 \%$, the highest percentage of prostheses on implants was made in the upper jaw, the type of prosthesis on implant with the highest percentage was the unitary prosthesis $58.78 \%$, according to the classification of edentulism the highest percentage was found in the partial edentulous, the The highest percentage in total edentulism was the hybrid prosthesis with $59.46 \%$ and in partial edentulism it was the unitary prosthesis with $66.80 \%$. Conclusions: Unitary prostheses were the most frequent, according to gender was female, according to San Isidro and according to RO service, according to the upper edentulous area, in total edentulous the hybrid prosthesis and in partial edentulous the unitary prosthesis.

\section{KEY WORDS: Edentulous arcade, toothless arcade, dental prosthesis, dental implants.}

\section{INTRODUCCIÓN}

En salud oral, el edentulismo es un estado que corresponde a la falta de una determinada cantidad de piezas dentarias en boca. Las causas pueden ser diversas, siendo las principales: la caries dental, la enfermedad periodontal y los traumatismos por lo que el odontólogo intenta sustituir los dientes perdidos por otros elementos que restauren la función y la estética $(1,2)$.

Se prevé que el cambio demográfico tendrá varias repercusiones en la salud oral, así se estima que el $70 \%$ de los pacientes habrá sufrido la pérdida de algún diente, por lo que el incremento de la esperanza de vida aumentará el riesgo de pérdida dentaria, estableciéndose una relación directamente proporcional entre la edad y el edentulismo (3).

La base de una correcta planificación es un correcto diagnóstico para que nos guíe y facilite el plan de tratamiento que se va a ejecutar en nuestros pacientes y también para sustentar nuestros tratamientos (1).

La sustitución de todos los dientes perdidos y estructuras adyacentes es un paso fundamental para el paciente desdentado consciente de su aspecto, por lo que la colocación de prótesis convencionales y prótesis sobre implantes son alternativas de tratamiento muy importantes en la vida de cualquier individuo (1).

Laaplicación originaldelos implantesosteointegrados, fue dar soporte a la prótesis de aquellos pacientes edéntulos que experimentaban problemas de movilidad de la misma (7). Posteriormente y tras el éxito obtenido, se extendió su aplicación a la restauración de ausencias unitarias o parciales y rehabilitaciones totales fijas $(8,9)$. Al analizar la satisfacción conseguida por el paciente, la ganancia de retención dejó de ser la única causa de rehabilitar sobre implantes, ganó importancia la estética, la función y la seguridad del paciente $(10,11)$.

La Clínica Dental Docente (CDD) de la Universidad Peruana Cayetano Heredia (UPCH) cuenta con servicios de posgrado en Rehabilitación Oral (RO) e Implantología Oral Integral (IOI) donde se atienden a pacientes con necesidades de prótesis convencionales y prótesis sobre implantes los cuales son planificados de acuerdo a las características de la zona edéntula, por lo que el propósito de este estudio fue determinar la frecuencia de tipos de tratamientos rehabilitadores sobre implantes durante los años 2016 y 2017.

El objetivo del presente trabajo fue determinar la frecuencia de tipos de prótesis sobre implantes planificados en pacientes edéntulos atendidos en 
los servicios del Posgrado de Rehabilitación Oral e Implantología Oral Integral en la Clínica Dental Docente de la Universidad Peruana Cayetano Heredia de enero del 2016 a diciembre del 2017.

También se determinaron las características de género, edad, sede y servicio de atención de los pacientes planificados con tratamientos de prótesis sobre implantes; el tipo de prótesis sobre implantes según zona edéntula y clasificación de edentulismo; el tipo de prótesis sobre implantes según características de género, edad, sede y servicio de atención; la zona edéntula según características de género, edad, sede y servicio de atención, y la clasificación de edentulismo según características de género, edad, sede y servicio de atención.

\section{MATERIAL Y MÉTODOS}

El presente estudio epidemiológico fue descriptivo, retrospectivo, observacional y transversal.

La población y muestra del estudio fue el mismo, se empleó una base de datos de todas las historias clínicas digitalizadas con tratamientos de prótesis sobre implantes con presentación de caso planificada y aceptada por el docente y paciente atendido en los servicios de Posgrado RO e IOI de la CDD de la Facultad de Estomatología desde enero 2016 a diciembre 2017.

Se incluyeron las historias clínicas realizadas en forma digitalizada de pacientes atendidos en los servicios de Posgrado RO e IOI de la CDD de la Facultad de Estomatología desde enero 2016 a diciembre 2017 con tratamientos de prótesis sobre implantes planificadas y aceptadas por el docente y paciente. Se excluyeron las historias clínicas con datos incompletos realizadas en forma digitalizada de tratamientos de prótesis sobre implantes durante los años 2016 y 2017 de la especialidades de RO e IOI. Aceptado el proyecto de investigación por el comité de ética con fecha 20-08-2018

Se depuró la base de datos de acuerdo con los tratamientos de prótesis sobre implantes, que cumplieron los criterios de inclusión. La recolección de datos se confeccionó en formato Excel y se realizó un conteo y clasificación de los tratamientos planificados, clasificándolos por tipo de prótesis sobre implantes, clasificación de edentulismo, zona edéntula, género, edad, sede y servicio. Se ordenaron los resultados según las variables de estudio para determinar las frecuencias. Para el análisis descriptivo se obtuvieron las frecuencias absolutas y relativas de las variables cualitativas, y promedio y desviación estándar de la variable cuantitativa. Para el análisis bivariado se aplicó para la variable cuantitativa las pruebas U de Mann Whitney y Kruskal Wallis y para variables cualitativas de empleó la prueba de Chi-cuadrado y Chi-cuadrado corregida por Yates. La presente investigación contó con un nivel de confianza de $95 \%$ y un $\mathrm{p}<0,05$, se usó el programa estadístico SPSS. v. 23.0.

\section{RESULTADOS}

Según la base de datos depurada la muestra fue de 296 pacientes planificados para recibir algún tipo de prótesis sobre implantes. El género con mayor porcentaje fue femenino con $68,58 \%$ (203 pacientes) y se encontró un 31,42\% (93 pacientes) en el sexo masculino. De acuerdo al grupo etáreo el promedio fue 54.23 años con desviación estándar de 13,81, con mayor porcentaje entre los 60 y 69 años $28,04 \%$ (83 pacientes) seguido por el grupo de 50 a 59 años $26,35 \%$ ( 78 pacientes) y el menor porcentaje fue entre los 80 a 89 años. En la sede San Isidro fue donde más tratamientos sobre implantes fueron planificados, $61,15 \%$ (181 pacientes) y en la sede San Martín se encontró 38,85\% (115 pacientes). RO fue el servicio con mayor de tratamientos planificados $60,81 \%(n=$ $180)$ y en IOI se encontró $39.19 \%$ (116 pacientes) (tabla 1). Según zona edéntula el mayor porcentaje de planificaciones fue en el maxilar superior con un $43,58 \%$ (129 pacientes), y en menor porcentaje en el maxilar superior e inferior $25 \%$ (74 pacientes). Y el tipo de prótesis sobre implante con mayor porcentaje fue la unitaria $58,78 \%$ (174 pacientes) y el de menor porcentaje fue la sobredentadura $4,73 \%$ (14 pacientes) siendo estadísticamente significativa $(p<0,01)$. Según clasificación de edentulismo el mayor porcentaje fue el edéntulo parcial $87,50 \%$ (259 pacientes), el mayor porcentaje fue la prótesis unitaria en el edéntulo parcial $66,80 \%$ (173 pacientes) y en el edéntulo total se planificó prótesis híbrida $59,46 \%$ (22 pacientes) seguido de la sobredentadura $37,84 \%$ (14 pacientes) siendo estadísticamente significativa $(\mathrm{p}<0,01)$ (tabla 2).

Según género en el sexo masculino se encontró 29,89\% (52 pacientes) prótesis unitaria, 19,23\% (10 
Tabla 1. Características de los pacientes atendidos con tratamientos de prótesis sobre implantes en los servicios del Posgrado de RO e IOI de la CDD de la UPCH, LimaPerú, 2016-2017.

\section{VARIABLES}

$\mathbf{n}$

$\%$

Género

$\begin{array}{lcc}\text { Masculino } & 93 & 31.42 \\ \text { Femenino } & 203 & 68.58 \\ & 54.23 & 13.81\end{array}$

$\operatorname{Edad}^{\circ}$

$\begin{array}{lcc}\text { De } 20 \text { a } 29 \text { años } & 19 & 6.42 \\ \text { De } 30 \text { a } 39 \text { años } & 31 & 10.47 \\ \text { De 40 a 49 años } & 50 & 16.89 \\ \text { De 50 a 59 años } & 78 & 26.35 \\ \text { De 60 a 69 años } & 83 & 28.04 \\ \text { De 70 a 79 años } & 30 & 10.14 \\ \text { De } 80 \text { a } 89 \text { años } & 5 & 1.69\end{array}$

Sede

$\begin{array}{lll}\text { San Martín } & 115 & 38.85 \\ \text { San Isidro } & 181 & 61.15\end{array}$

Servicio

$\begin{array}{lcc}\text { Rehabilitación O. } & 180 & 60.81 \\ \text { Implantología } & 116 & 39.19 \\ \text { Oral } & & \\ & 296 & 100.00\end{array}$

Total

100.00

$\mathrm{n}$ : Frecuencia absoluta.

$\%$ : Frecuencia relativa.

○Se calculó el promedio y desviación estándar por ser variable cuantitativa. pacientes) prótesis múltiple, 21,43\% (3 pacientes) sobredentadura, 59,26\% (16 pacientes) prótesis híbrida y 41,38\% (12 pacientes) con prótesis unitaria y múltiple. Mientras que en el sexo femenino se encontró 70,11\% (122 pacientes) prótesis unitaria, $80.77 \%$ (42 pacientes) prótesis múltiple, 78,57\% (11 pacientes) sobredentadura, 40,74\% (11 pacientes) prótesis híbrida y 58,62\% (17 pacientes) unitaria y múltiple (p 0,02). Según edad todos los promedios encontrados fueron estadísticamente significativos $(\mathrm{p}<0,01)$. En la prótesis unitaria el promedio fue de 50,85 años con una desviación estándar de 14,19, el mayor porcentaje de prótesis tipo unitaria se encontró entre los 60 a 69 años 26,44\% $(n=46)$, prótesis tipo múltiple el promedio fue de 56,02 años con desviación estándar de 9,91, el mayor porcentaje de prótesis tipo múltiple se encontró entre los 50 a 59 años de $34,62 \%$ (18 pacientes), prótesis tipo sobredentadura el promedio fue de 67,64 años con desviación estándar de 13,57 , el mayor porcentaje fue entre los 70 a 79 años 42,86\% (6 pacientes), prótesis tipo híbrida el promedio fue de 65,19 años con desviación estándar de 9.28, el mayor porcentaje fue entre los 60 a 69 y 70 a 79 años 33,33\% (9 pacientes) para ambos y con prótesis tipo unitaria y múltiple el promedio fue de 54,59 años con desviación estándar de 11,75, el mayor porcentaje fue entre los 50 a 59 y los 60 a 69 años con un 27,59\% (8 pacientes) para ambos; siendo estadísticamente significativa $(\mathrm{p}<0,01)$.

Según sede, en San Martín se planificó 38.51\% (67 pacientes) de prótesis tipo unitaria, 42,31\% (22

Tabla 2. Tipo de prótesis sobre implantes según zona edéntula y clasificación de edentulismo de los pacientes atendidos en los servicios del Posgrado de RO e IOI de la CDD de la UPCH, Lima-Perú, 2016-2017.

\begin{tabular}{|c|c|c|c|c|c|c|c|c|c|c|c|c|c|c|}
\hline \multirow{3}{*}{$\begin{array}{l}\text { Tipo de Prótesis } \\
\text { sobre Implantes }\end{array}$} & \multirow{3}{*}{$\mathbf{n}$} & \multirow{3}{*}{$\%$} & \multicolumn{6}{|c|}{ Zona Edéntula } & \multirow{3}{*}{$\mathbf{P}$} & \multicolumn{5}{|c|}{ Clasificación de Edentulismo } \\
\hline & & & \multicolumn{2}{|c|}{ Superior } & \multicolumn{2}{|c|}{ Inferior } & \multicolumn{2}{|c|}{$\begin{array}{l}\text { Superior e } \\
\text { inferior }\end{array}$} & & \multicolumn{2}{|c|}{$\begin{array}{c}\text { Edéntulo } \\
\text { total }\end{array}$} & \multicolumn{2}{|c|}{$\begin{array}{c}\text { Edéntulo } \\
\text { parcial }\end{array}$} & \multirow[t]{2}{*}{$\mathbf{p}$} \\
\hline & & & $\mathbf{n}$ & $\%$ & $\mathbf{n}$ & $\%$ & $\mathbf{n}$ & $\%$ & & $\mathbf{n}$ & $\%$ & $\mathbf{n}$ & $\%$ & \\
\hline Unitaria & 174 & 58.78 & 91 & 70.54 & 50 & 53.76 & 33 & 44.59 & & 1 & 2.70 & 173 & 66.80 & \\
\hline Múltiple & 52 & 17.57 & 20 & 15.50 & 16 & 17.20 & 16 & 21.62 & & 0 & 0.00 & 52 & 20.08 & \\
\hline Sobre dentadura & 14 & 4.73 & 2 & 1.55 & 11 & 11.83 & 1 & 1.35 & $<0.01 *$ & 14 & 37.84 & 0 & 0.00 & $<0.01 *$ \\
\hline Híbrida & 27 & 9.12 & 10 & 7.75 & 10 & 10.75 & 7 & 9.46 & & 22 & 59.46 & 5 & 1.93 & \\
\hline Unitaria y múltiple & 29 & 9.80 & 6 & 4.65 & 6 & 6.45 & 17 & 22.97 & & 0 & 0.00 & 29 & 11.20 & \\
\hline Total & 296 & 100.00 & 129 & 43.58 & 93 & 31.42 & 74 & 25.00 & & 37 & 12.50 & 259 & 87.50 & \\
\hline
\end{tabular}


Balarezo Razzeto J, Díaz Sarabia E, Brignardello Cedrón F, Paz Mayurí C, Santos Escalante C, Ronquillo Herrera W, Leon Manco R, Quintana del Solar M.

Tabla 3. Tipo de prótesis sobre implantes según características de los pacientes atendidos en los servicios del Posgrado de RO e IOI de la CDD de la UPCH, Lima-Perú, 2016-2017.

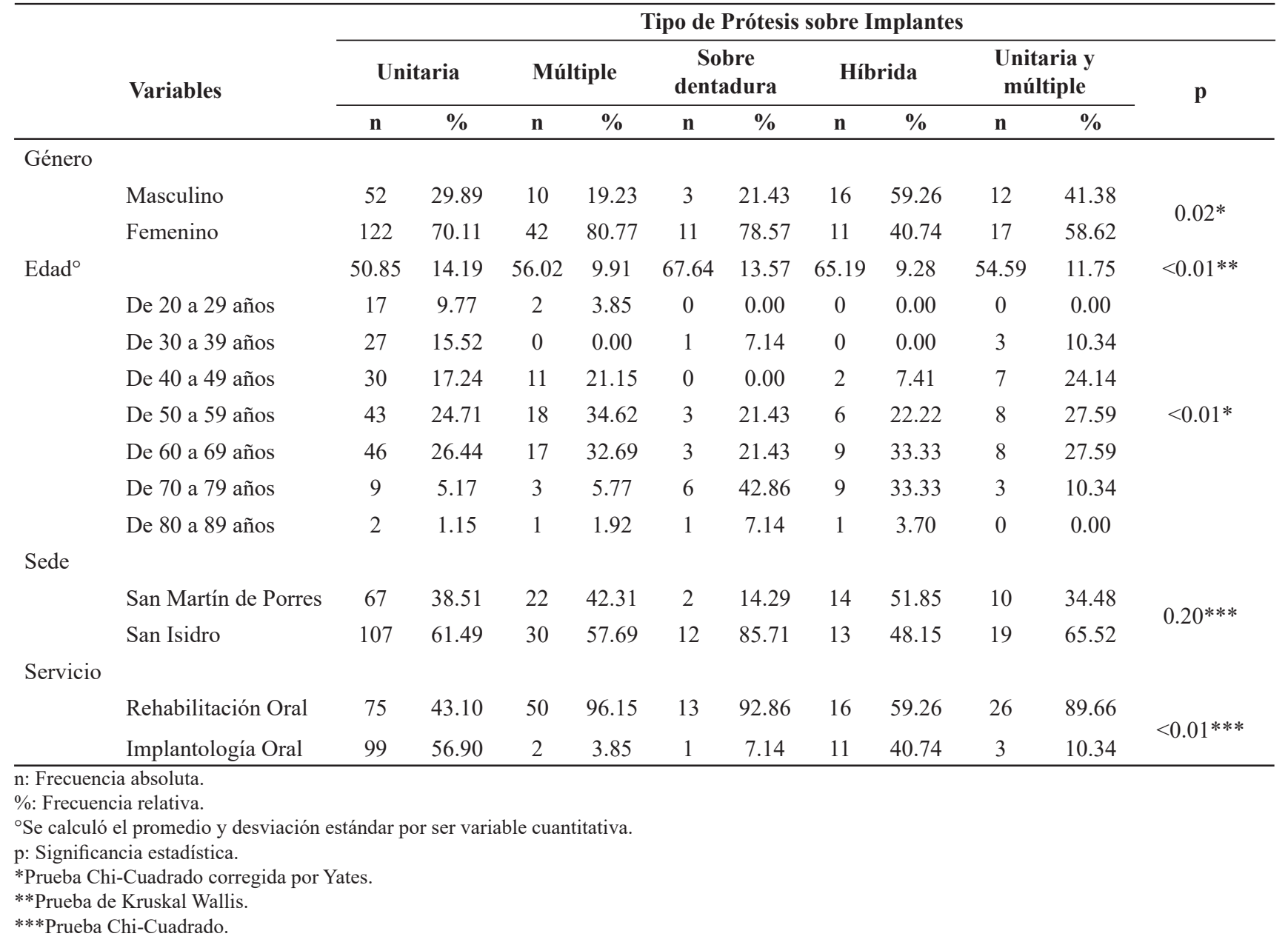

pacientes) prótesis tipo múltiple, $14,29 \%$ (2 pacientes) prótesis tipo sobredentadura, $51.85 \%$ (4 pacientes) prótesis tipo híbrida y $34,48 \%$ (10 pacientes) prótesis tipo unitaria y múltiple. En la sede San Isidro se encontró un $61,49 \%$ (107 pacientes) de prótesis tipo unitaria, 57,69\% (30 pacientes) prótesis tipo múltiple, $85.71 \%$ (12 pacientes) prótesis tipo sobredentadura, $48,15 \%$ (13 pacientes) prótesis tipo híbrida y $65,52 \%$ (19 pacientes) prótesis tipo unitaria y múltiple ( $\mathrm{p} 0,20)$. Según servicio, en el área de RO se encontró $43,10 \%$ (75 pacientes) de prótesis tipo unitaria, 96,15\% (50 pacientes) con prótesis tipo múltiple, 92,86\% (13 pacientes) con prótesis tipo sobredentadura, 59,26\% (16 pacientes) con prótesis tipo híbrida y 89,66\% (26 pacientes) con prótesis tipo unitaria y múltiple. En la sede San Isidro se encontró 56,90\% (99 pacientes) de prótesis tipo unitaria, $3,85 \%$ ( 2 pacientes) prótesis tipo múltiple, $7,14 \%$ (1 paciente) prótesis tipo sobredentadura, $40,74 \%$ (11 pacientes) prótesis tipo híbrida y 1,34\% (3 pacientes) prótesis tipo unitaria y múltiple, siendo estadísticamente significativa ( $p<0,01$ ). Según servicio la mayor frecuencia de coronas unitarias fueron planificadas en el servicio de IOI con 56,90\% (99 pacientes) a diferencia de los otros tipos de prótesis que fueron en su mayoría en RO prótesis múltiple $96,15 \%$ (50 pacientes), prótesis tipo sobredentadura $92,86 \%$ (13 pacientes), prótesis híbrida 59,26\% (16 pacientes) y la combinación de prótesis unitaria y múltiple con $89,66 \%$ (26 pacientes), estadísticamente significativa $(\mathrm{p}<0,01)$ (tabla 3 ).

Según género se encontró en la zona edéntula superior un mayor porcentaje en el sexo femenino $69,77 \%$ $(n=90)$ mientras en el sexo masculino $30.23 \%$ $(n=39)$, en la zona edéntula inferior se encontró también para el sexo femenino el mayor porcentaje $65,59 \%(n=61)$ y $34,41 \%(n=32)$ para el sexo masculino y en la zona edéntula superior e inferior 
se dio de igual manera en el sexo femenino un mayor porcentaje $70,27 \%(\mathrm{n}=52)$ y $29,73 \%(\mathrm{n}=22)$ para el sexo masculino (p 0,75). Según edad los valores promedios según zona edéntula superior encontrados fueron 52,33 con desviación estándar de 14,54, zona edéntula inferior 55,26 con desviación estándar de 14,57 y zona edéntula superior e inferior 56,23 con una desviación estándar de 10,99 con un (p 0.12). El mayor porcentaje en la zona edéntula superior se encontró entre los 60 a 69 años de edad $24,03 \%$ $(\mathrm{n}=31)$, y el menor porcentaje entre los 80 a 89 años de edad 2,33\% (n=3). En la zona edéntula inferior se encontró el mayor porcentaje entre los 60 a 69 años de edad $31,18 \%(n=29)$, y el menor porcentaje entre los 80 a 89 años de edad $2,15 \%(n=2)$. Y en la zona edéntula superior e inferior se encontró el mayor porcentaje entre los 50 a 59 años de edad 32,43\% $(\mathrm{n}=24)$, y el menor porcentaje entre los 80 a 89 años de edad con un $0,00 \%(n=0)(p 0,21)$. Según sede el mayor porcentaje en la zona edéntula superior se encontró en la sede de San Isidro con un 57,36\% $(\mathrm{n}=74)$, y el menor porcentaje en la sede de Martín de
Porres 42,64\% $(n=55)$. En la zona edéntula inferior se encontró el mayor porcentaje también en la sede de San Isidro $62,37 \%(n=58)$, y el menor porcentaje en la sede de Martín de Porres 37,63\% ( $n=35)$. Y en la zona edéntula superior e inferior se dio de igual manera en la sede de San Isidro el mayor porcentaje $66,22 \%(\mathrm{n}=49)$, y el menor porcentaje se dio en la sede de Martín de Porres con un 33,78\% ( $\mathrm{n}=25)$ ( $\mathrm{p}$ 0,44 ). Según servicio el mayor porcentaje en la zona edéntula superior se planificó en el área de RO con un $55.81 \%(\mathrm{n}=72)$, y el menor porcentaje en el área de IOI $44,19 \%(n=57)$. En la zona edéntula inferior se encontró el mayor porcentaje también en el área de RO 64,52\% ( $\mathrm{n}=60)$, y el menor porcentaje en el área de IOI $35,48 \%(n=33)$. Y en la zona edéntula superior e inferior se dio de igual manera en el área de RO el mayor porcentaje $64.86 \%(n=48)$, y el menor porcentaje en el área de IOI con un $35,14 \%(n=26)$ (p 0,30) (tabla 4).

Según género en el edéntulo total el mayor porcentaje se encontró en el sexo femenino 59,46\% (22 pacientes)

Tabla 4. Zona edéntula según características de los pacientes atendidos en los servicios del Posgrado de RO e IOI de la CDD de la UPCH, Lima-Perú, 2016-2017.

\begin{tabular}{|c|c|c|c|c|c|c|c|c|}
\hline & \multirow{3}{*}{ Variables } & \multicolumn{6}{|c|}{ Zona Edéntula } & \multirow{4}{*}{$\mathbf{P}$} \\
\hline & & \multicolumn{2}{|c|}{ Superior } & \multicolumn{2}{|c|}{ Inferior } & \multicolumn{2}{|c|}{ Superior e inferior } & \\
\hline & & $\mathbf{n}$ & $\%$ & $\mathbf{n}$ & $\%$ & n & $\%$ & \\
\hline \multicolumn{8}{|l|}{ Género } & \\
\hline \multirow{10}{*}{ Edad $^{\circ}$} & Masculino & 39 & 30.23 & 32 & 34.41 & 22 & 29.73 & \multirow{2}{*}{$0.75 *$} \\
\hline & Femenino & 90 & 69.77 & 61 & 65.59 & 52 & 70.27 & \\
\hline & & 52.33 & 14.54 & 55.26 & 14.57 & 56.23 & 10.99 & $0.12 * *$ \\
\hline & De 20 a 29 años & 9 & 6.98 & 9 & 9.68 & 1 & 1.35 & \multirow{7}{*}{$0.21 *$} \\
\hline & De 30 a 39 años & 18 & 13.95 & 8 & 8.60 & 5 & 6.76 & \\
\hline & De 40 a 49 años & 27 & 20.93 & 10 & 10.75 & 13 & 17.57 & \\
\hline & De 50 a 59 años & 29 & 22.48 & 25 & 26.88 & 24 & 32.43 & \\
\hline & De 60 a 69 años & 31 & 24.03 & 29 & 31.18 & 23 & 31.08 & \\
\hline & De 70 a 79 años & 12 & 9.30 & 10 & 10.75 & 8 & 10.81 & \\
\hline & De 80 a 89 años & 3 & 2.33 & 2 & 2.15 & 0 & 0.00 & \\
\hline \multicolumn{9}{|l|}{ Sede } \\
\hline & San Martín de Porres & 55 & 42.64 & 35 & 37.63 & 25 & 33.78 & \multirow{2}{*}{$0.44^{*}$} \\
\hline & San Isidro & 74 & 57.36 & 58 & 62.37 & 49 & 66.22 & \\
\hline \multicolumn{9}{|l|}{ Servicio } \\
\hline & Rehabilitación Oral & 72 & 55.81 & 60 & 64.52 & 48 & 64.86 & \multirow{2}{*}{$0.30^{*}$} \\
\hline & Implantología Oral & 57 & 44.19 & 33 & 35.48 & 26 & 35.14 & \\
\hline
\end{tabular}


Tabla 5. Clasificación de edentulismo según características de los pacientes atendidos en los servicios del Posgrado de RO e IOI de la CDD de la UPCH, Lima-Perú, 2016-2017.

\begin{tabular}{|c|c|c|c|c|c|c|}
\hline & \multirow{3}{*}{ Variables } & \multicolumn{5}{|c|}{ Clasificación de Edentulismo } \\
\hline & & \multicolumn{2}{|c|}{ Edéntulo total } & \multicolumn{2}{|c|}{ Edéntulo parcial } & \multirow{2}{*}{$\mathbf{P}$} \\
\hline & & $\mathbf{n}$ & $\%$ & $\mathbf{n}$ & $\%$ & \\
\hline \multicolumn{7}{|l|}{ Género } \\
\hline & Masculino & 15 & 40.54 & 78 & 30.12 & \multirow{2}{*}{$0.20 *$} \\
\hline & Femenino & 22 & 59.46 & 181 & 69.88 & \\
\hline \multirow[t]{8}{*}{ Edad $^{\circ}$} & & 64.86 & 11.95 & 52.71 & 13.40 & $<0.01 * *$ \\
\hline & De 20 a 29 año & 0 & 0.00 & 19 & 7.34 & \multirow{7}{*}{$<0.01 * * *$} \\
\hline & De 30 a 39 años & 2 & 5.41 & 29 & 11.20 & \\
\hline & De 40 a 49 años & 2 & 5.41 & 48 & 18.53 & \\
\hline & De 50 a 59 años & 8 & 21.62 & 70 & 27.03 & \\
\hline & De 60 a 69 años & 11 & 29.73 & 72 & 27.80 & \\
\hline & De 70 a 79 años & 12 & 32.43 & 18 & 6.95 & \\
\hline & De 80 a 89 años & 2 & 5.41 & 3 & 1.16 & \\
\hline \multicolumn{7}{|l|}{ Sede } \\
\hline & San Martín de Porres & 14 & 37.84 & 101 & 39.00 & \multirow{2}{*}{$0.89 *$} \\
\hline & San Isidro & 23 & 62.16 & 158 & 61.00 & \\
\hline \multicolumn{7}{|c|}{ Servicio } \\
\hline & Rehabilitación Oral & 26 & 70.27 & 154 & 59.46 & \multirow[b]{2}{*}{$0.21 *$} \\
\hline & $\begin{array}{l}\text { Implantología Oral } \\
\text { Integral }\end{array}$ & 11 & 29.73 & 105 & 40.54 & \\
\hline \multicolumn{7}{|c|}{$\begin{array}{l}\text { n: Frecuencia absoluta. } \\
\text { \%: Frecuencia relativa. } \\
\text { 'Se calculó el promedio y desviación estándar por ser variable cuantitativa. } \\
\text { p: Significancia estadística. } \\
\text { *Prueba Chi-Cuadrado. } \\
\text { **Prueba de U de Mann Whitney. } \\
\text { ***Prueba Chi-Cuadrado corregida por Yates. }\end{array}$} \\
\hline
\end{tabular}

y en sexo masculino $40,54 \%$ (15 pacientes) y en el edéntulo parcial también el mayor porcentaje se dio en el sexo femenino 69,88\% (181 pacientes) y en sexo masculino se encontró 30,12\% (78 pacientes) (p $0,20)$. Según edad todos los promedios encontrados fueron estadísticamente significativos $(\mathrm{p}<0,01)$ con un promedio de 64,86 y una desviación estándar de 11.95 para el edéntulo total y con un promedio de 52,71 y una desviación estándar e 13.40 para el edéntulo parcial. En el edéntulo total el mayor porcentaje se encontró entre las edades de 70 a 79 años 32.43\% (12 pacientes), seguido por las edades entre los 60 a 69 años $29,73 \%$ (11 pacientes) y entre 50 a 59 años $21,62 \%$ (8 pacientes) y en el edéntulo parcial el mayor porcentaje se encontró entre los 60 a 69 años 27,80\% (72 pacientes), seguido por las edades entre los 50 a 59 años $27,03 \%$ (70 pacientes) y entre 40 a 49 años 18,53\% (48 pacientes); siendo estadísticamente significativa $(\mathrm{p}<0,01)$. Según sede el edéntulo total el mayor porcentaje se encontró en San Isidro 62,16\% (23 pacientes) y el menor porcentaje en San Martín 37,84\% (14 pacientes). En el edéntulo parcial se encontró el mayor porcentaje también en la sede San Isidro 61,00\% (158 pacientes), menor porcentaje en sede San Martín de 39,00\% (101 pacientes) (p 0,89). Según servicio en el edéntulo total el mayor porcentaje se encontró en el área de RO 70,27\% (26 pacientes) y el menor porcentaje en IOI 29,73\% (11 pacientes). En el edéntulo parcial se encontró también el mayor porcentaje en el área de RO 59,46\% (154 pacientes), y el menor porcentaje en IOI 40,54\% (105 pacientes) (p 0,21)(tabla 5).

\section{DISCUSIÓN}

El presente estudio tuvo como finalidad determinar las frecuencias de los diversos tipos de tratamientos rehabilitadores sobre implantes planificados según 
las características de los pacientes edéntulos parciales y totales atendidos en los servicios de RO e IOI de la CDD de la UPCH por lo que fue necesario el estudio para poder aportar datos estadísticos y con ello mejorar y contribuir el acceso a servicios de salud oral con tratamientos de prótesis sobre implantes de las personas adultas, ayudando así en el desempeño académico-clínico de los servicios de RO e IOI.

De un total de 296 pacientes con diversos tipos de prótesis sobre implantes que fueron planificados en los servicios de RO e IOI; se encontró más del doble de pacientes de género femenino, que el masculino por lo que en su mayoría son los pacientes de género femenino que acuden y se planifica la mayoría de prótesis sobre implantes y notamos que el tipo de prótesis no está relacionado al sexo, coincidiendo con datos reportados en el estudio de Betanncourt (13) sobre la mayor prevalencia de la colocación de implantes en pacientes mujeres que acudieron al quirófano de la facultad de odontología de la universidad central del ecuador durante los años lectivos 2008 - 2009,2009 - 2010 y 2010 - 2011.

Haikola (17) y colaboradores mencionan que el edentulismo es un fenómeno multifactorial y su prevalencia global está disminuyendo, pero que está va aumentando en los grupos de mayor edad. Y en nuestro estudio en el cual a los pacientes se les planificó las rehabilitaciones sobre implantes el rango de edad encontrado fue de 20 a 89 años con un promedio de 54.23 años encontrándose el mayor porcentaje entre los 60 y 69 años de edad y los resultados nos muestran que de acuerdo a la edad en los grupos de 20 hasta los 69 años iba en aumento al igual que el estudio de Haikola. Además en el presente estudio se observó una relación estadísticamente significativa entre la edad y los distintos tipos de prótesis sobre implantes. Con respecto a las sedes, en San Isidro se encontró en mayor número la frecuencia de prótesis sobre implantes planificadas que en SMP. Este Hallazgo puede deberse a su ubicación dentro de Lima Metropolitana y a la situación socio-económica de las personas.

En relación con los servicios se encontró con un porcentaje mayor en el servicio de RO comparado con el servicio de IOI, y también en el servicio de RO se realizó mayormente rehabilitaciones múltiples o completas comparativamente al servicio de IOI, asumimos que esto se debe a que en el servicio de RO atienden pacientes edéntulos parciales y totales en los cuales le realizan tratamientos integrales también con prótesis convencionales como las prótesis completas, prótesis parcial removible, prótesis fija y combinación de estas, pudiendo a la vez en el mismo paciente planificar utilizando prótesis sobre implantes a diferencia del servicio de IOI que atienden solo pacientes más específicos para tratamientos con prótesis sobre implantes, además también contribuye que la cantidad de operadores es mayor en el servicio de RO que en IOI. Por lo que el servicio de RO es quien planifica mayor cantidad de prótesis sobre implantes.

Según la clasificación del edentulismo en nuestro estudio se encontró mayor número de edéntulos parciales que totales y también encontramos una relación significativa con respecto a la edad en el edéntulo parcial y total. Clásicamente, la pérdida de dientes ha sido uno de los estados dentales más prevalentes entre los pacientes adultos. En el Perú, Balarezo (19) en 1988 en el Cono Norte de Lima Metropolitana registró un $74.3 \%$ de los examinados habían perdido al menos una pieza dentaria y que el edentulismo parcial es más frecuente que el edentulismo total. Torres (22) en un estudio de prevalencia de pacientes desdentados totales bimaxilares donde se aprecia que la incidencia de edentulismo se da más a partir de edades entre $50 \mathrm{y}$ 60 años. Mientras que en nuestro estudio se encontró en el edéntulo total para la planificación de prótesis sobre implantes el mayor porcentaje se encuentra entre las edades de 70 a 79 años y tener en cuenta que el edentulismo se presenta en la mayoría de los adultos a nivel mundial y está relacionado al nivel socio-económico $(15,16)$.

Con respecto a los tipos de prótesis sobre implantes la frecuencia fue mayor para confección de coronas unitarias que rehabilitaciones completas, debido a que los pacientes atendidos eran en su mayoría edéntulo parciales y también probablemente por el desconocimiento de estas alternativas por parte de la población o por el alto costo del tratamiento.

En cuanto a la zona edéntula se confeccionaron mayor número de prótesis en el maxilar superior casi el doble que el inferior, por lo que la maxila es la más frecuente en ser rehabilitada con prótesis sobre 
implantes, coincidiendo con un estudio realizado por Valverde (23) en el 2011, detalló que en el maxilar superior se encontró mayor edentulismo que el inferior. En cuanto a las limitaciones de nuestro estudio fue el diseño, ya que el realizado fue de corte transversal y no longitudinal, el cual analizaría mejor la frecuencia de necesidad y situación de las prótesis sobre implantes con el transcurso del tiempo.

En cuanto a las limitaciones de nuestro estudio fue el diseño, ya que el realizado fue de corte transversal y no longitudinal, el cual analizaría mejor la frecuencia de necesidad y situación de las prótesis sobreimplantes con el transcurso del tiempo.

Este estudio nos ha permitido determinar cuál es la planificación de los tratamientos rehabilitadores sobre implantes más predominantes con la finalidad de conocer una información que permita tener un control estadístico de las pacientes atendidos para estructurar mejor su correcto abordaje. Asimismo cual es la demanda de estos tratamientos protésicos en los servicios de RO e IOI y de esta forma mejorar el equipamiento y la capacitación del personal para la rehabilitación oral protésica del edéntulo parcial o total y cubrir satisfactoriamente las necesidades de los pacientes atendidos en la CDD de la UPCH con un adecuado desempeño académico-clínico.

Los autores declaran no tener conflictos de interés.

\section{CONCLUSIONES}

En el presente estudio se encontró un total de 296 pacientes planificados con diversos tipos de prótesis sobre implantes en pacientes edéntulos parciales y totales en la CDD en los servicios de Posgrado en RO e IOI de la UPCH y se concluye que:

Con mayor frecuencia se planificaron prótesis unitarias, prótesis múltiples, combinación de prótesis unitaria y múltiple, prótesis híbridas $\mathrm{y}$ sobredentaduras.

Según género fue mayor en el sexo femenino, duplicando al masculino, según edad entre los 50 a 69 años con un promedio de 54 años, según sede fue mayor en San Isidro que en San Martín y según servicio se planificaron mayormente en RO que IOI.

Según zona edéntula fue la prótesis unitaria, lo más frecuente en edéntulos totales fue la prótesis híbrida y en edéntulos parciales fue la prótesis unitaria.

Según género fue mayor en el sexo femenino excepto en las prótesis híbridas, según edad las prótesis unitarias, múltiples y la combinación de estas se encontró mayormente entre los 50 a 69 años, mientras que las prótesis tipo sobredentaduras en pacientes entre los 70 a 79 años y las prótesis híbridas entre los 60 a 79 años de edad, según sede mayor frecuencia en la sede San Isidro a excepción de las prótesis híbridas que fue mayor en la sede San Martín, según servicio la mayor frecuencia de coronas unitarias fue en el servicio de IOI a diferencia de los otros tipos de prótesis que fueron en su mayoría en $\mathrm{RO}$.

Las zonas edéntulas más frecuentes se presentaron en el maxilar superior, según género fue mayor en el sexo femenino, según edad entre los 50 y 69 años de edad, según sede la mayor frecuencia fue en la sede de San Isidro y en cuanto a los servicios el de mayor frecuencia fue RO.

La clasificación de edentulismo según género en los edéntulos totales y parciales fue más frecuente el sexo femenino, según edad el edéntulismo total fue mayor entre los 60 y 79 años en tanto que en el edéntulismo parcial fue entre los 50 y 69 años, según sede la frecuencia de edéntulos totales y parciales fue mayor en San Isidro y según servicio fue mayor en RO.

\section{Correspondencia:}

Jose Antonio Balarezo Razzeto

Correo Electrónico : jose.balarezo@upch.pe

\section{REFERENCIAS BIBLIOGRÁFICAS}

1. Balarezo JA. Prótesis sobre implantes en el edéntulo total. Lima: Editorial Savia; 2014.

2. López O J. Prevalencia de Edentulismo Parcial Según la Clasificación de Kennedy en el servicio de Rehabilitación Oral del Centro Médico Naval Cirujano Mayor Santiago Távara. Tesis Título Profesional de Cirujano Dentista. Lima; Universidad Mayor de San Marcos; 2009.

3. Marcus SE, Drury TF, Brown LJ, Zion GR. Tooth retencion and tooth loss in the permanent dentition of adults: United States, 1988-1991. J Dent Res 1996; 75: 684-95.

4. Panasiuk L, Kosiniak-Kamysz W, Horoch A, Paprzycki P, Karwat D. Tooth loss among adult rural and urban inhabitants of the Lublin Region. Ann Agric Environ 
Med. 2013; 20(3): 637-41.

5. Loza D. Prótesis parcial removible. Caracas: Actualidades Médico Odontológicas Latinoamérica; 1992.

6. Branemark PI, Hansson BO, et al. Osseointegrated implants in the treatment of the edentulous jaw. Experience from a 10-year period. Scand J Plast Reconstruct Surg. 1977; 16: 1-132.

7. Van Steenberghe D, Lekholm U, Bolender C, et al. Applicability of osseointegrated oral implants in the rehabilitation of partial edentulism: a prospective multicenter study on 558 fixtures. Int J Oral Maxillofac Implants. 1990; 5(3): 272-81.

8. Van Steenberghe D, Klinge B, Linden U, Quirynen M, Herrmann I, Garpland C. Periodontal indices around natural and titanium abutments: a longitudinal multicenter study. J Periodontol. 1993; 64(6): 538-41.

9. Preciado A, Del Río J, Suárez-García MJ, Montero J, Lynch CD, Castillo-Oyagüe R. Differences in impact of patient and prosthetic characteristics on oral healthrelated quality of life among implant-retained overdenture wearers. J Dent. 2012; 40(10): 857-65.

10. Geertman ME, Slagter AP, van Waas MA, Kalk W. Comminution of food with mandibular implantretained overdentures. J Den Res. 1994; 73(12): 185864.

11. Craddock H, Youngson C, Manogue M, Blance A. Occlusal changes following posterior tooth loss in adults. Part 1: a study of clinical parameters associated with the extent and type of supraeruption in unopposed posterior teeth. Journal of Prosthodontics. 2007; 16 (6): 485-94.

12. Díaz PG. Factores asociados al edentulismo en pacientes diagnosticados en la Clínica de la Facultad de Odontología UNMSM. Tesis bachiller. Lima: Universidad Nacional Mayor de San Marcos; 2009.

13. Betanncourt E, Prevalencia de la colocación de implantes en pacientes mujeres que acudieron al quirófano de la facultad de odontología de la universidad central del ecuador durante los años lectivos 2008 - 2009,2009 - 2010 y 2010 - 2011. 2015.Tesis para obtener el Título de Odontóloga Quito: Universidad Central de Ecuador; 2011.

14. Cooper LF. The current and future treatment of edentulism. J Prosthodont. 2009; 18:116-22.
15. Petersen PE, Yamamoto T. Improvingthe oral health Programme community Dent Oral Epidemiol. 2005; 33: 84-92.

16. Saman D, Lemieux A, Arevalo O, Lutfiyya M. A population-based study of edentulism in the US: does depression and rural residency matter after controlling por potential cofounders? BMC Public Health. 2014; 14 (1): 65.

17. Haikola B, Oikarinen K, Söderholm AL, Remes-Lyly T, Sipilä K. Prevalence of edentulousness and related factors among elderly Finns. J Oral Rehabil. 2008; 35(11):827-35.

18. Kratochvil FJ. Maintaining supporting structures with a removable partial prosthesis. Journal Prosthet Dent. 1971; 25: 167-74.

19. Balarezo J. Relación entre el nivel socioeconómicocultural y la situación de la salud bucal en pobladores de zonas urbano marginales del Cono Norte de LimaMetropolitana. Prevalencia de pérdida dentaria deciduas, permanentes y determinación de necesidades protésicas. Tesis de Bachiller en Estomatología. Lima: Universidad Peruana Cayetano Heredia; 1986.

20. Raygada J. Perfil de salud bucal y necesidades de tratamiento en una comunidad adulta rural de la sierra central del Perú. Tesis bachiller. Lima: Universidad Peruana Cayetano Heredia. Facultad de Estomatología; 1988.

21. Dolan T, Gilbert G, Duncan R, Foerster U. Risk indicators of edentulism, partial tooth loss and prosthetic status among black and white middle-aged and older adults. Community Dent Oral Epidemiol. 2001; 29 (5):329-340.

22. Torres D, Prevalencia de pacientes desdentados totales bimaxilares. Tesis para obtener el Título de Odontóloga. Guayaquil, Ecuador: Universidad de Guayaquil; 2017.

23. Valverde HR. Relación del edentulismo parcial con interferencias oclusales en pacientes del Servicio de Rehabilitación Oral del Post Grado de la Facultad de Estomatología Roberto Beltrán de la Universidad Peruana Cayetano Heredia, 2008-2010. Tesis maestría. Lima: Universidad Peruana Cayetano Heredia; 2011.

24. Winkelman R, Orth K. Implantes dentales. Barcelona: Espax Editores; 1994. p. 35-9.

25. Bert M, Missika A. Implantes osteointegrados. Barcelona: Masson Editores; 1993. p. 25-8. 